\title{
Genetic diversity of rare iris species in the Southern Urals
}

Mikhaylova E.V. ${ }^{1 *}$, Mustafina A.N. ${ }^{2}$, Kryukova A.V. ${ }^{2}$

${ }^{1}$ Institute of Biochemistry and Genetics, UFRC RAS, Ufa, Russia

${ }^{2}$ South Ural Botanical Garden, UFRC RAS, Ufa, Russia

*e-mail:mikhele@list.ru

Iris scariosa and I. pumila are two rare species of iris found in the steppes of the Southern Urals. Both of them are included in the Red Data Book of the Russian Federation. I. pumila is an ancestor of many modern cultivars, and I. scariosa is known for its drought and salt tolerance and can also be used in breeding. The Republic of Bashkortostan and Orenburg oblast are situated on the eastern border of I. pumila habitat and western border of I. scariosa habitat, so Southern Urals is the area where these two species coexist. Therefore, it is important to study these unique populations, their morphological and genetic diversity and possibilities of hybridization. We studied DNA samples from 29 populations of iris discovered in Southern Urals using different RAPD and ISSR primers. We observed not only phenotypical, but also genetic interspecific and interpopulation variability. Moreover, several samples distinguished as I. scariosa on the basis of external features, genetically showed higher similarity to I. pumila and might be hybrids.

Acknowledgements: This work was supported by the RFBR grant mol_a 18-34-00022. 